\section{Die Bildung eines rotbraunen Pigmentes durch Mikroorganismen bei Anwesenheit von Trypto- phan in der Nährlösung}

Verschiedene Mikroorganismen scheiden in die Nährlösung ein rotbraunes Pigment aus, wenn ihnen Tryptophan als Stickstoffquelle geboten wird ${ }^{1-3}$. Versuche mit mehreren Phycomyceten- und Ascomyceten-Arten ergaben bei 14 von 20 geprüften Stämmen Pigmentbildung bei Anwesenheit von Tryptophan im Milieu (Tabelle I). Jeder Organismus wurde zur Kontrolle des Wachstums mit der in der Originalnährlösung ${ }^{1,4-6}$ angegebenen Stickstoffquelle, mit der Hälfte hievon und einer entsprechenden Menge Tryptophan sowie nur mit Tryptophan als Stickstoffquelle kultiviert. Die Stickstoffkonzentration in den Nährlösungen betrug einheitlich $10^{-2} M / 1$.

Da sich eine tryptophanhaltige Nährlösung beim Sterilisieren $\left(115^{\circ} \mathrm{C} / 15 \mathrm{~min}\right)$ leicht bräunlich färbt, wurde in allen Versuchen das Tryptophan in Wasser gelöst getrennt sterilisiert und nachher der sterilisierten Nährlösung aseptisch zugefügt. Die so hergestellte Nährlösung ist völlig farblos und klar. Sie färbt sich bei längerem Stehen (bis 14 Tage) unter Kulturbedingungen $\left(29^{\circ} \mathrm{C}\right.$, dunkel) leicht bräunlich, etwas stärker unter Belichtung (Fluoreszenzröhren Philips TL 40 W/33, 3000 Lux). Organismen, deren Nährlösung nur die Farbintensität steriler Kontrollen annahm, wurden in Bezug auf ihr Pigmentbildungsvermögen als negativ und nur solche als positiv beurteilt, bei welchen eine kräftige Braunfärbung beobachtet wurde.

Aus Rhizopus oryzae-Kulturflüssigkeit ${ }^{5}$ kann das Pigment mit organischen Lösungsmitteln extrahiert werden (Tabelle II). $n$-Butanol und Cyclohexanol extrahieren nur unvollständig. Im Butanolextrakt lässt sich papierchromatographisch Tryptophan nachweisen. Neben dem Tryptophanfleck enthält das Chromatogramm keine mit Ninhydrin, Ehrlich's Reagens ${ }^{7}$ und Nitrit + Naphthylamin $^{7}$ reagierenden Substanzen. Das Pigment selbst ver-

Tabelle I

\begin{tabular}{lll}
\hline Organismus & $\begin{array}{l}\text { Nähr- } \\
\text { lösung }\end{array}$ & $\begin{array}{l}\text { Pigment- } \\
\text { bildunga }\end{array}$ \\
\hline
\end{tabular}

Phycomycetes

Mucor hiemalis Wehmer

M. ramannianus Möller

Phycomyces blakesleanus Burgeff

Rhizopus arrhizus Fischer

$R$. bovinus v. Beyma

$R$, chinensis Saito

$R$. circinans v.'Tiegh.

$R$. nigricans Ehrenbg.

$R$. nodosus Namysl.

$R$. oryzàe W. et Pr. G.

$R$. pusillus Naoumoff

$R$. pygmaeus Naoumoff

$R$. suinus Nielsen

$R$. tritici Saito

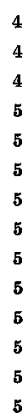

Ascomycetes

Aspergillus niger v. Tiegh.

Neurospora crassa Shear et Dodge

Penicillium candidum Link.

$P$. chrysogenum Thom.

$P$. notatum Westl.

Rhodotorula rubra (Demme) Lodder

$\begin{array}{ll}\mathbf{4} & ++ \\ \mathbf{4} & - \\ \mathbf{4} & - \\ \mathbf{5} & ++ \\ \mathbf{5} & - \\ \mathbf{5} & ++ \\ \mathbf{5} & ++ \\ \mathbf{5} & - \\ \mathbf{5} & ++ \\ \mathbf{5} & ++ \\ \mathbf{5} & ++ \\ \mathbf{5} & ++ \\ \mathbf{5} & ++ \\ \mathbf{5} & ++ \\ & \\ \mathbf{4} & + \\ \mathbf{6} & + \\ \mathbf{1} & ++ \\ \mathbf{1} & - \\ \mathbf{1} & ++ \\ \mathbf{4} & ++ \\ & ++\end{array}$

a - Nicht stärker als in unbeimpften Kontrollen; ++ sehr kräftig. schwindet während des Chromatographierens (Laufmittel: Butanol-Essigsäure-Wasser $4: 1: 1$ ) und ist auf dem entwickelten Chromatogramm nicht mehr zu lokalisieren. Aus dem Phenol-Chloroformextrakt fällt nach Zugabe von vier Volumteilen Äther ein Niederschlag aus, welcher sich papierchromatographisch wie Tryptophan verhält. Das Phenol-Chloroformgemisch kann im Vakuum bei $50^{\circ} \mathrm{C}$ unter Durchleiten von Stickstoff abdestilliert werden. Der Rückstand löst sich in Alkohol mit brauner Farbe und enthält kein Tryptophan mehr.

Das Pigment ist gegen Ansäuerung der Nährlösung mit Essig- und Salzsäure beständig. Versetzt man Nährlösung mit konzentrierter Salzsäure im Verhältnis $1: 1$, so schlägt die Farbe von braun in purpur um. Neutralisation mit fester Natronlauge lässt wieder die braune Farbe erscheinen. Der Farbumschlag kann wiederholt werden. Mit Cyclohexanol kann das Pigment auch in der purpurnen Form extrahiert werden ${ }^{8}$.

Tabelle II

\begin{tabular}{ll}
\hline Extraktionsmittel & Resultat \\
\hline Ather $^{a, b}$ & sehr geringes Extraktionsver- \\
mögen & keine Extraktion \\
Athylmethylketon & keine Extraktion \\
Benzin $^{\mathrm{a}}$ & keine Extraktion \\
Benzol $^{\mathrm{a}}$ & gutes Extraktionsvermögen, \\
n-Butanol & Extraktion unvollständig \\
& keine Extraktion \\
Chloroforma & wie $n$-Butanol \\
Cyclohexanol & geringes Extraktionsvermögen \\
Essigsäureäthylester $b$ & keine Extraktion \\
$n$-Hexan & vollständige Extraktion \\
Phenol-Chloroform $1: 1(\mathrm{w} / \mathrm{v})$ & keine Extraktion \\
Petroläther $\left(70^{\circ} \mathrm{C}, 110 / 120^{\circ} \mathrm{C}\right)$ & keine Extraktion \\
Tetrachlorkohlenstoff & keine Extraktion \\
Xylol &
\end{tabular}

a Kein Unterschied, ob Nährlösung sauer (pH ca. 3) oder mit $\mathrm{Na}_{2} \mathrm{CO}_{3}$ auf $\mathrm{pH} 8$ gebracht. ${ }^{\mathrm{B}}$ Bei Extraktion nach ${ }^{2}$ wird sowohl durch Ather als auch durch Athylacetat ein sehr geringer Teil des Pigmentes extrahiert. Die Restnährlösung bleibt dunkelbraun gefärbt, mit $n$-Butanol kann weiter extrahiert werden.

Summary. Several Phycomycetes and Ascomycetes form a reddish-brown pigment if their nutrient medium contains tryptophan. The pigment can be extracted from the medium with $n$-butanol, cyclohexanol, and a mixture of phenol and chloroform $1: 1(\mathrm{w} / \mathrm{v})$.

\section{H. ТнӧNI}

Pflanzenphysiologisches Institut der Universität

Bern (Schweiz), 2. Februar 1966.

1 P. Schwarze und O. Frandsen, Planta 50, 353 (1958).

2 F. Hageman n, Arch. Mikrobiol. 49, 150 (1964).

3 M. Polster und M. Svobodova, Experientia 20, 637 (1964).

4 R. FluRI, Arch. Mikrobiol. 33, 195 (1959).

5 H. Thöni, Arch. Mikrobiol. 46, 338 (1963).

6 G. W. Beadle, J. biol. Chem. 156, 684 (1944).

7 H. Hellmann, Z. physiol. Chem. 287, 205 (1951).

8 Dr. K. H. Erismann danke ich für wertvolle Hinweise und für die Bereitstellung von Material und Hilfsmitteln des Institutes.

9 Gegenwärtige Adresse: Statistical Laboratory and Department of Statistics, Iowa State University of Science and Technology, Ames, Iowa 50010, USA. 